\title{
Lingual nerve retraction increases the risk of temporary lingual nerve damage during mandibular third molar surgery
}

\author{
Is there any difference in sensory disturbance of the lingual nerve after \\ mandibular third molar surgery with or without lingual flap retraction?
}

\section{Gomes AC, Vasconcelos BC, de Oliveira e Silva ED, da Silva LC. Lingual nerve damage after mandibular third molar surgery: $a$ randomized clinical trial. J Oral Maxillofac Surg 2005; 63:1443- 1446}

Design A split-mouth randomised controlled trial (RCT) was conducted in two university hospitals in Brazil.

Intervention Patients, referred for bilateral mandibular third molar removal, were randomly allocated to have the procedure performed either on one side (experimental group) with lingual flap retraction or the opposite side without (control group). The procedure was carried out under local or general anaesthesia. Local anaesthesia was also given to all patients under general anaesthesia. Ostectomy to remove buccal bone was performed in all cases, after the buccal flap was raised.

Outcome measure Sensory disturbance was evaluated after $24 \mathrm{~h}$ and on postoperative day 7 by an oral and maxillofacial surgeon who was blind to the operation procedure. A pin-prick test was used to confirm nerve injury and to classify the sensory disturbance (anaesthesia, hypoaesthesia, paraesthesia or dysaesthesia).

Results A total of 55 patients were recruited. The proportion of individuals with sensory disturbance was higher in those operated upon under general/ local anaesthesia (13.8\%) than in patients operated upon under local anaesthesia only (3.8\%), although the difference was not significant. Lingual nerve sensation disturbance occurred in $9.1 \%$ of the experimental group, whereas no cases were recorded in the control group. This difference was statistically significant $(P<0.001)$ as measured by the Cochran test. Of five cases where sensory disturbance was registered, four were classified as paraesthesia and one as hypoaesthesia. All cases were classified as temporary disturbance because after 3 months after the operation there was spontaneous recovery.

Conclusions Lingual nerve retraction represented a risk factor to temporary lingual nerve damage during mandibular third molar surgery.

\section{Commentary}

This trial is clearly focused on a particular population, on one intervention and on a complication that is of importance. Assignment of subjects to treatment groups was randomised, although the method of randomisation is not given. Follow up (at $24 \mathrm{~h}$ and 7 days) was standardised and complete. Assessors were blind to treatment assignment.

It is not possible to know whether the groups were similar at the start of the trial in terms of age, gender, social class and education status, however, an omission that would have been avoided if the trial had been reported according to CONSORT guidelines, ${ }^{1}$ and neither is the treatment effect size reported. Furthermore, a pretrial calculation of sample size does not appear to have been done. In fact, the sample size was sufficient to identify between-group differences in nerve damage not severe enough to produce lingual anaesthesia, but it was not large enough to detect differences between rates of anaesthesia.

Despite the methodological and presentational shortcomings, the message from this trial is clear: lingual retraction during the surgical removal of lower third molars increases rather than decreases the risk of lingual nerve damage. Furthermore, since the characteristics of third molar impaction and disease are universal, the results of this RCT are relevant to all practitioners around the world who perform this procedure. Importantly, lingual nerve retraction has not been advocated for purposes other than lingual nerve 'protection' which means that avoiding this technique imposes no extra burdens on clinicians nor risks for patients. Note that the authors cite the other principal RCT of this surgical intervention ${ }^{2}$ but not the later systematic review.

\section{Practice point}

- Lingual nerve retraction should be avoided during surgical removal of mandibular third molars.

\section{Jonathan P Shepherd}

Department of Oral Surgery, Medicine and Pathology, University of Wales College of Medicine, Cardiff, Wales, UK

1. Moher D, Schulz KF, Altman D. The CONSORT statement: revised recommendations for improving the quality of reports of parallel-group randomised trials. J Am Med Assoc 2001; 285:1987-1991.

2. Robinson PP, Smith KG. Lingual nerve damage during lower third molar removal: a comparison of two surgical methods. Br Dent J 1996; 180:456-461.

3. Pichler JW, Beirne OR. Lingual flap retraction and prevention of lingual nerve damage associated with third molar surgery: a systematic review of the literature. Oral Surg Oral Med Oral Pathol Oral Radiol Endod 2001; 91:395-401.

Evidence-Based Dentistry (2006) 7, 47.

doi:10.1038/sj.ebd.6400402 